\title{
The irresistible biology of resistin
}

\author{
Gökhan S. Hotamisligil \\ Division of Biological Sciences and Department of Nutrition, Harvard School Of Public Health, Boston, Massachusetts, USA \\ J. Clin. Invest. 111:173-174 (2003). doi:10.1172/JCI200317605.
}

Fuel sources have historically triggered many battles throughout the globe and will likely continue to do so. The design of biological systems is no different. Adipose tissue holds the richest energy sources and exerts substantial influence on the organism. While this fundamental truth has eluded scientists for a long time, it is now clear that adipocytes have established effective communication networks and gained control over important trade routes to maintain their influential status. To this end, these innocent-looking cells appear to utilize two main tools: fatty acids and polypeptide hormones. It is likely that they have additional, yet unknown, signaling mechanisms.

It has been known for some time that obesity is associated with insulin resistance and diabetes. Yet how and why these problems are so closely linked at the molecular level has not been clear. As in most such cases, lack of knowledge led to many conspiracy theories - my favorite one being that agents produced by fat cells affect metabolic control at other sites. An example of such an agent is the inflammatory cytokine TNF- $\alpha$, which is produced in excess in obesity and causally linked to insulin resistance (1). Many other such agents have now been added to this list, although unlike TNF- $\alpha$, some do not mediate adverse effects on

\footnotetext{
Address correspondence to: Gökhan S. Hotamisligil, Division of Biological Sciences and Department of Nutrition, Harvard School Of Public Health, 665 Huntington Avenue, Boston, Massachusetts 02115, USA. Phone: (617) 432-1950; Fax: (617) 432-1941; E-mail: ghotamis@hsph.harvard.edu.

Conflict of interest: The author has declared that no conflict of interest exists.

Nonstandard abbreviations used: thiozolidinedione (TZD); resistin-like molecule (RELM).
}

metabolism. In fact, some, such as leptin and adiponectin, work to improve the metabolic status of their fellow organs (2).

\section{A role for resistin in obesity and insulin resistance}

The most recent addition to this adverse family of adipocyte-derived polypeptides is resistin. During a search for targets of thiozolidinediones (TZDs), a class of insulin sensitizers, Steppan et al. discovered that expression of the gene encoding this secreted protein was suppressed by TZD treatment in adipocytes (3). Subsequently, additional members of this family, resistin-like molecule- $\alpha$ (RELM $\alpha)$ and RELM $\beta$, and alternative biological activities were reported $(4,5)$. In their original report, Steppan et al. showed that levels of this protein in the blood of obese mice are increased and that TZDs acted to suppress this obesityrelated increase in resistin expression (4). Moreover, in vivo administration of recombinant resistin to otherwise normal mice caused insulin resistance, whereas administration of an antiresistin antibody increased insulin sensitivity of obese and insulin-resistant animals. Finally, cultured adipocytes exposed to resistin showed a reduction of insulin-stimulated glucose uptake, whereas the antiresistin antibody produced the opposite effect (4). This discovery generated much interest and excitement, not only as a mechanism of action for this class of drugs but also as a potential general mechanism by which obesity is linked to insulin resistance and diabetes, not to mention the potential therapeutic applications of the discovery.

Yet it became rapidly clear that the story might be much more complicated than originally envisioned. First, expression of resistin mRNA and protein was found to be strongly suppressed in adipose tissues in many models of experimental obesity (6-8). This raised the question of why expression of a protein that mediates insulin resistance would be strongly suppressed in conditions of insulin resistance. Could protein levels in obesity still be elevated in the circulation due to increased adipose tissue mass? This issue was also recently addressed, and it appears that serum protein levels in experimental obesity, for example in $\mathrm{db} / \mathrm{db}$ mice, are significantly reduced when compared to those in lean control mice, similarly to resistin mRNA and protein levels in adipose tissue (9). In addition, treatment of animals with insulinsensitizing drugs such as metformin or several different categories of synthetic PPAR- $\gamma$ ligands, including TZDs, resulted in a complex and inconsistent pattern of resistin expression $(3,6-8,10)$. On the other hand, agents that cause insulin resistance, such as TNF- $\alpha$, negatively regulate expression and secretion of resistin in cultured adipocytes (11). Finally, unlike in the mouse gene, the human homologue of resistin was not detected in human adipocytes in any appreciable amount, and the quantities of mRNA found in adipose tissue were not found to be related to obesity or diabetes (12). To complicate things further, one of the two homologues of resistin, RELM $\alpha$, is absent from the human genome.

\section{Resistin and RELM $\beta$ impair glucose metabolism in the liver}

This picture is certainly complicated. In this issue of the JCI, Rajala et al. provide much-needed clarification of the biological functions of this family of proteins (13). In an elegant set of in vivo experiments, the authors 